\title{
General conditions for railway wheel polygonal wear to evolve
}

\author{
Bo Peng 1,2, , Simon Iwnicki ${ }^{1}$, Philip Shackleton ${ }^{1}$, Yang Song ${ }^{1}$ \\ ${ }^{1}$ Institute of Railway Research, University of Huddersfield, Huddersfield, UK \\ ${ }^{2}$ CRRC Zhuzhou Locomotive Co.,Ltd. Zhuzhou, China \\ *E-mail: bo.peng@vip.163.com
}

\begin{abstract}
This paper reports fundamental research on the general conditions for railway wheel polygonal wear to evolve. A common workflow for prediction of railway wheel polygonization is presented including assumptions, simulation scheme, and wear models. Based on this workflow, some rules for the evolution of railway wheel polygonization are proposed providing innovative perspectives to understand the basic mechanism of railway wheel polygonization. After summarizing these rules, the general conditions for railway wheel polygonal wear to evolve are established. The phase between the instantaneous wear depth and the excitation is the key indicator determining the wheel OOR (Out-Of-Roundness) evolution direction (to grow or to diminish). The evolution tendency curve obtained from the instantaneous wear FRF (Frequency Response Function) is a useful tool to predict the OOR evolution, especially for predicting the OOR order that would grow predominantly at a given speed. If one or more structural modes can dominate the evolution tendency curve, and the energy distribution of track excitation allows this/these structural modes to be excited effectively, corresponding OOR orders can occur dominantly.
\end{abstract}

Keywords: Railway wheel polygonization; polygonal wear; general conditions; evolution mechanism.

\section{Introduction}

The phenomenon of wheel polygonization has been widely found in all kinds of rolling stock for decades, including high-speed trains, metro trains, and locomotives. It introduces periodic excitation in the wheel-rail interface causing harmful vibration to the vehicle-track system. The consequences can be noise, reduction of comfort, component damage, and track deterioration [1]. To ensure the safety and comfort of railway rolling stocks, the most common passive solution is either shortening the maintenance period for wheels, which can lead to significant increase of maintenance costs, or changing the vehicle speed regularly, which clearly adds complexity to the operation management. To address the root-cause of this problem, its mechanism has to be understood so that some effective solutions can be proposed.

Currently it appears that railway wheel polygonization must be a fixed-frequency issue. Specifically, there must be some structural modes of the vehicle/track dynamic system that can be excited to initiate the wheel polygonization with particular orders at particular speeds. Based on this view, researchers are continuing efforts to find the structural mode frequency that can match the dominant vibration frequency of wheels in the presence of wheel OOR, either by measurement or simulation. Some potential influencing factors have been revealed, such as vehicle speed, P2 resonance [2,3], wheelset flexibility [4], local rail flexibility [5], wheelset imbalance [6], material hardness [7], wheel flat [8], AC motor [9], and so on. However, there has not been a universal explanation that can cover all circumstances, possibly because the railway wheel polygonization is a multi-factor problem, and for different scenarios (e.g. vehicle type, track type, speed range) the wheel polygonization may be attributed to 
different combination of those potential factors, which makes the problem hard to be explained. Consequently, it is necessary to carry out fundamental research on the basic evolution rules for railway wheel polygonization. As it is not easy to carry out this kind of fundamental research through experiment due to the high experiment cost and serious influence on the normal operation, simulation becomes the best alternative but the reasonability and robustness of the prediction program need to be guaranteed.

Compared to the wheel transverse wear prediction which has been successfully applied in businesses, the prediction of railway wheel polygonization is far less mature up to now. As the characteristics of the wheel circumferential wear are essentially different from those of the wheel transverse wear, the simulation method cannot be just copied from one to another, and some adaptions have to be made, although both of them follow an iterative loop program structure. Few researches have elaborated on the prediction program of railway wheel polygonization on itself in a comprehensive sense. Johansson [3], Morys [10], and Meinke [6] are some pioneer researchers who introduced the iterative loop program structure to the prediction of railway wheel polygonization. $\mathrm{Fu}$ [11] discussed some details of the numerical simulation for polygonal wear. The authors [12] have also proposed a practical method for simulation of railway wheel polygonization with illustration of the programme structure. To better understand the complex phenomena of railway wheel polygonization, the prediction program has to be logical and consistent to provide a reasonable result that can reflect the reality.

This work is carrying out fundamental research to investigate the general conditions for railway wheel polygonal wear to evolve. A common workflow for predicting the railway wheel polygonization is firstly introduced, including some assumptions, the simulation scheme, and the wear models. Based on this workflow, several rules for the evolution of railway wheel polygonization are proposed providing innovative perspectives to understand the basic mechanism. By summarizing these rules, the general conditions for wheel OOR to evolve are established.

\section{Common workflow for prediction of railway wheel polygonization}

\subsection{Assumptions}

The most realistic simulation of the wheel tread wear is in $3 \mathrm{D}$ and real-time sense, which means that the 3D geometry of the wheel tread should change for every wheel revolution. However, this is either technically impossible or unbearably time-consuming with today's technology. As a result, similar to the simulation of railway wheel transverse profile wear, some assumptions have to be made to allow the simulation of the wheel circumferential wear to be feasible. Some common assumptions are listed below:

- The wheel transverse profile is assumed not to change, with the focus only on the change in the wheel circumferential profile. This assumption is justified by the fact that, when railway wheel polygonization occurs, the circumferential shapes measured at different wheel lateral positions are quite similar $[13,14]$.

- The instantaneous wear is assumed to be equally distributed cross the transverse direction, which is implicit from the first assumption. For each contact patch, an averaged wear depth is calculated.

- Only the abrasive wear mechanism is accounted for, implying that the wear only occurs in the sliding zone of the contact patch. A common combination is Hertz \& FASTSIM, while other types of contact model could also be applied. 
- The nominal wheel radius can be assumed not to change or reduce with wear. If the radius is reducing during the iteration, the simulation workflow will be more complicated. In this paper, the nominal wheel radius is assumed not to change.

Under these assumptions, the mean value of the instantaneous wear depth that must be considered for wheel transverse wear estimation is no longer needed, and it is only the fluctuation of the instantaneous wear that will determine the evolution of wheel polygonization.

\subsection{Simulation scheme}

Almost all of the existing prediction programs for wheel wear follow a closed-loop simulation scheme. Compared to the wheel transverse profile prediction, the simulation of polygonal wear has its particularities. A general loop simulation scheme for railway wheel polygonization is illustrated in Figure 1, which can be divided into 4 steps.
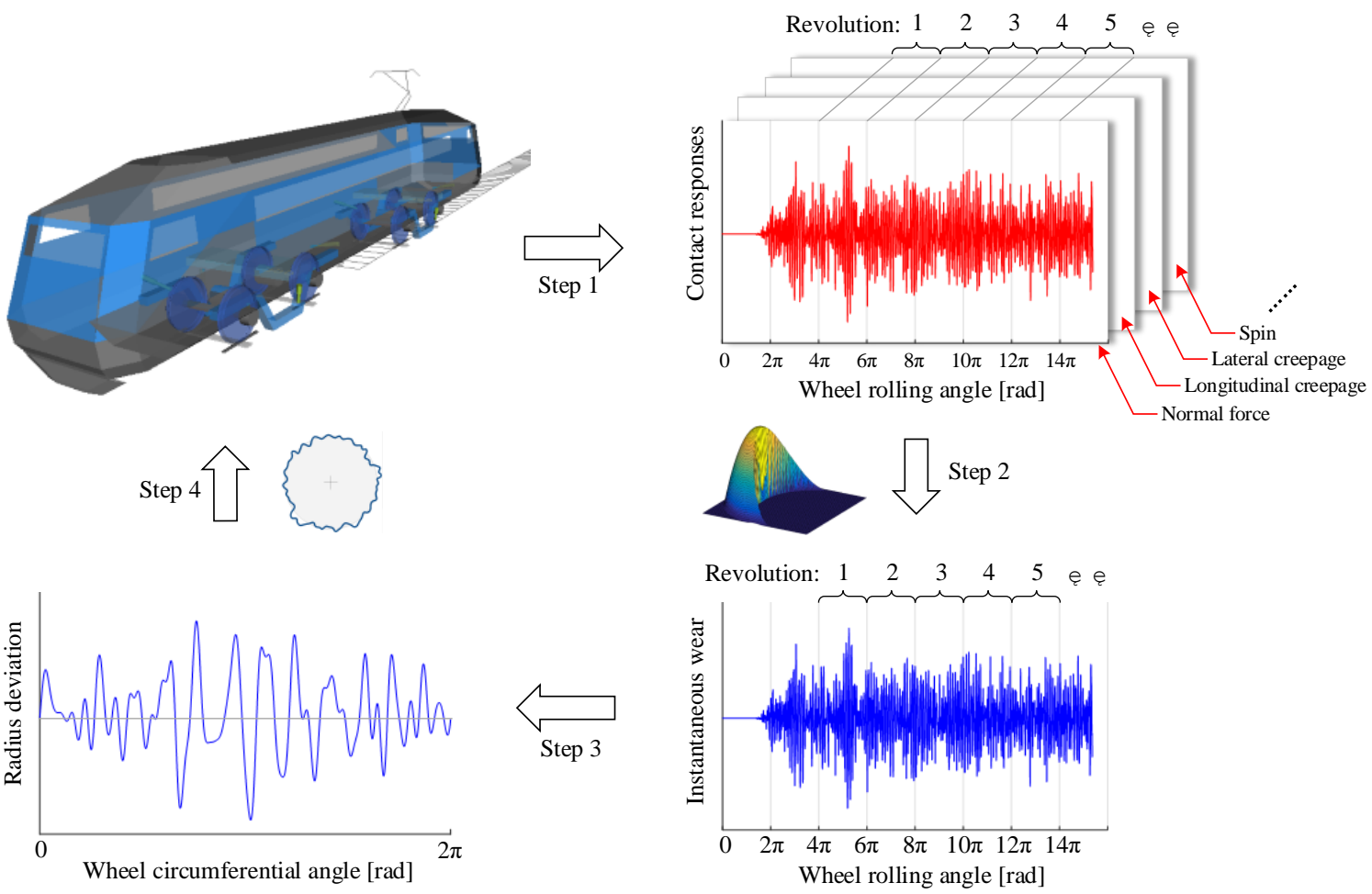

Figure 1. Loop simulation scheme for railway wheel polygonization

Step 1: The Multi-Body System (MBS) simulation is responsible for calculating the contact responses for the wear estimation. If the KTH (Royal Institute of Technology) wear model [15] is used, nine contact parameters are needed: normal force, creepages (longitudinal, lateral, and spin), contact patch size (semi-axis a and b), Kalker coefficients (C11, C22, and C23).

Step 2: The $\mathrm{x}$-coordinate of all the contact responses is replaced by the wheel rolling angles in order to facilitate the calculation of the polygonal wear for each wheel revolution, as well as the accumulation of the circumferential wear of multiple wheel revolutions. The instantaneous wear depth is calculated in real-time so that the wheel circumferential shape is allowed to evolve. Under this circumstance, the wear model used here is actually no longer a 'long-term' wear model, but a 'short-term' wear model instead. 
Step 3: The instantaneous wear depth is first interpolated, so as to be equally distributed for every circle (4096 points for one circle in this paper), and then accumulated and averaged by a number of wheel revolutions to obtain the polygonal wear abound the wheel circumference for one iteration. The minimum number of wheel revolutions required for a convergent result is scenario dependent, this will be discussed in Section 3.4.

Step 4: The wheel circumferential shape is updated for the next MBS simulation. The newly generated OOR should be smoothed, filtered to exclude high frequencies that are either not of interest or are generated by artificial spikes, and ensured to have a continuity from the start to the end as the OOR is a cyclic closed curve. These three tasks can be completed simultaneously with a modified Fourier Series method. Finally, the new OOR is scaled with an amplification coefficient to represent a much larger number of revolutions. There is not a specific criteria for the amplification coefficient, but only a compromise between the computing accuracy and efficiency.

\subsection{Wear models}

An improved understanding has been obtained on the applicability of existing popular wear models for simulation of railway wheel polygonization by carrying out a comprehensive comparative study [16] between four representative wear models developed by BRR (British Rail Research) [17], KTH (Royal Institute of Technology) [15], USFD (University of Sheffield) [18], and Professor Zobory [19], respectively. Although the wear models investigated are different from each other in their original definition, they actually share the same nature in calculating the instantaneous wear depth, as all of them can be analytically converted into a uniform expression with a uniform Wear Index (WI). Results show that all the wear models present a similar ability to reflect the fluctuation of the instantaneous wear under various circumstances. As a result, the selection from among these wear models does not matter significantly, but the wear coefficient adopted is the key to obtaining a meaningful result for a real case. More details can refer to [16]. This paper employs the KTH wear model to calculate the instantaneous wear depth, while similar results can be obtained as well with other wear models.

\section{Some rules for evolution of railway wheel polygonization}

It is generally believed that the railway wheel polygonization is a fixed-frequency issue. According to the simulation, this fixed frequency could come either directly from the excitation to the vehicle-track dynamic system, or from some modal resonance that can be triggered and excited effectively by excitation to fluctuate the instantaneous wear in the same modal frequency. The excitation to the vehicle/track dynamic system can either be the track excitation (rail irregularity, sleeper passing oscillation, and so on) or the wheel OOR on itself, if the motor vibration and the aerodynamics are not considered. In the real case, the polygonal wear must be gradually formed under excitation of both track excitation and newly developed OOR simultaneously during the evolution, which behaves as a complicated process. To make this complex issue easier to understand, a single wheelset model is intentionally adopted to expound some basic rules and features that the instantaneous wear will follow. Simpack is used as the MBS package. A wheelset of a locomotive with 23 tonne axle load is adopted as the research object. A co-running track model with sleeper following the wheelset is used to simply represent the track flexibility. Guidance forces with linear stiffness and damping in three axes are applied at both sides of the wheelset axle, representing the dynamic coupling effect from the bogie frame. The main parameters of the model are listed in Table 1. 
Table 1 Main model parameters

\begin{tabular}{lll}
\hline Main parameters & Value & Unit \\
\hline Wheelset mass & 3562 & $\mathrm{~kg}$ \\
Radius & 0.625 & $\mathrm{~m}$ \\
Preload on each primary suspension & 105 & $\mathrm{kN}$ \\
Longitudinal stiffness of primary suspension & $3.6 \mathrm{e} 7$ & $\mathrm{~N} / \mathrm{m}$ \\
Lateral stiffness of primary suspension & $4.8 \mathrm{e} 6$ & $\mathrm{~N} / \mathrm{m}$ \\
Vertical stiffness of primary suspension & $2.9 \mathrm{e} 6$ & $\mathrm{~N} / \mathrm{m}$ \\
Vertical damping of primary suspension & $4 \mathrm{e} 4$ & $\mathrm{Nm} / \mathrm{s}$ \\
Sleeper mass & 308 & $\mathrm{~kg}$ \\
Vertical stiffness of ballast & $5.4 \mathrm{e} 8$ & $\mathrm{~N} / \mathrm{m}$ \\
Vertical damping of ballast & $1.5 \mathrm{e} 5$ & $\mathrm{Ns} / \mathrm{m}$ \\
\hline
\end{tabular}

\subsection{The basic rule for initial OOR to evolve}

Assuming that the wheel has an initial OOR with a single harmonic order (the wheel circumference can be exactly divided by the wavelength), and the track is perfect without any irregularities, when the wheel is running at a constant speed, there will be a cyclic excitation caused by this initial OOR with a single frequency according to Equation (1).

$$
\text { Frequency=Speed/Wavelength }
$$

All contact responses as well as the instantaneous wear depth will be fluctuated with the same excitation frequency, but can have different phases. As a result, the updating of the wheel circumferential shape after one wheel revolution comes down to a simple mathematical problem illustrated as Equation (2).

$$
\mathrm{A} \cdot \sin (f x)-\mathrm{B} \cdot \sin (f x+\phi)=\sqrt{\mathrm{A}^{2}-2 \mathrm{AB} \cos \phi+\mathrm{B}^{2}} \cdot \sin \left(f x+\arctan \frac{-\mathrm{B} \sin \phi}{\mathrm{A}-\mathrm{B} \cos \phi}\right)
$$

Where: A is the fluctuation amplitude of initial OOR, B the fluctuation amplitude of instantaneous wear depth, $f$ the excitation frequency, $\phi$ the phase of instantaneous wear depth with respect to initial OOR, and $x$ the wheel rolling angle. Note that the mean value of OOR will always be subtracted, and the focus is only put on the fluctuation part. So the growth ratio of OOR can be expressed as:

$$
\text { Growth ratio }=\frac{\sqrt{\mathrm{A}^{2}-2 \mathrm{AB} \cos \phi+\mathrm{B}^{2}}}{\mathrm{~A}}=\sqrt{(\mathrm{B} / \mathrm{A})^{2}-2(\mathrm{~B} / \mathrm{A}) \cos \phi+1}
$$

The growth ratio can be plotted as a 3D figure with $\mathrm{B} / \mathrm{A}$ and $\phi$ as two individual variables, as shown in Figure 2. Having a look at the lower 3D sub-figure in Figure 2, the surface above the pink plane (ratio=1) indicates that the OOR will grow, otherwise the OOR will diminish. Although the growth ratio is determined by B/A and $\phi$ together, in reality $\mathrm{B} / \mathrm{A}$ is extremely small for one wheel revolution, meaning that the situation can only occur in the dashed red region, where the phase $\phi$ is the only factor determining whether the OOR will grow or not. By zooming in this region and switching to the front view, the relationship between the phase $\phi$ and the growth ratio of OOR can be presented in the upper sub-figure (assuming $B / A=1 e-5$ ). If the phase between the instantaneous wear depth and the initial OOR is within $90^{\circ}$ to $270^{\circ}$ range, the initial OOR will grow. At 180 degree, the growth rate reaches to the maximum. Although the growth ratio is at a very low order of magnitude for one wheel 
revolution, the effective growth ratio will be amplified with running distance, and then the change of the wheel circumferential shape can be notable.

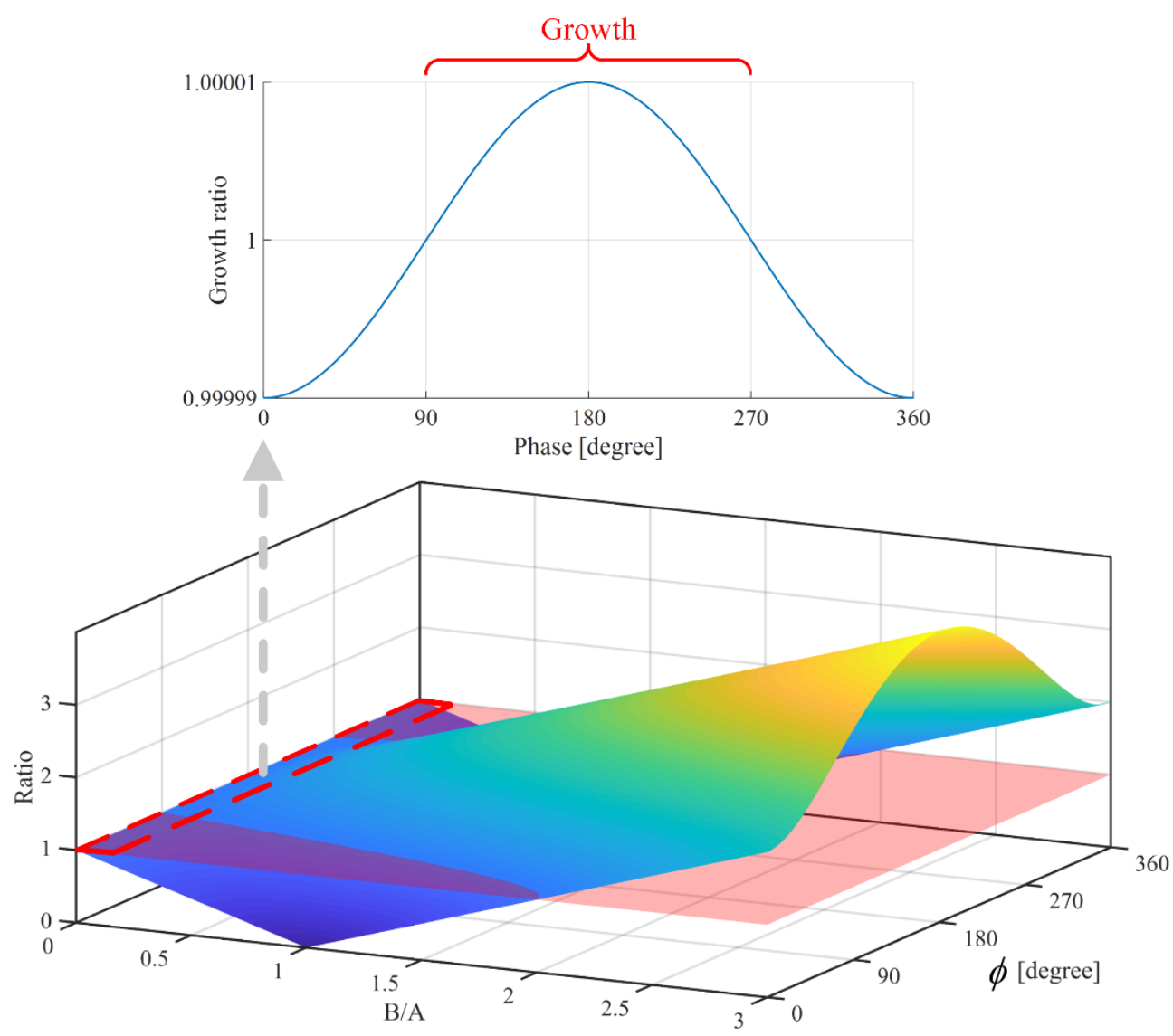

Figure 2. Growth ratio against $\mathrm{B} / \mathrm{A}$ and $\phi$

\subsection{The effect of excitation on contact parameters}

The excitation position of OOR and rail irregularity is illustrated in Figure 3, as they are applied in Simpack. The OOR changes the nominal radius of the wheel to vary the wheel profile only in vertical direction, while the rail irregularity alters the position of the rail profile in both vertical and lateral directions. Under excitation, the position of the contact patch will be searched for every time step accordingly, and all contact parameters will be varied correspondingly. Theoretically, if a single-frequency harmonic excitation of OOR or vertical rail irregularity is applied, all contact responses will fluctuate with the same excitation frequency. But the phase of different contact parameters against the excitation can be different from each other, and the phase of contact parameters caused by OOR and vertical rail irregularity respectively is also different from each other, which presents as a very complicated phenomena that is hard to interpret.

Figure 4 shows an example of the phase of some contact parameters against a harmonic excitation of OOR and vertical rail irregularity respectively. For this example, the scenario is set the same for OOR and vertical rail irregularity to facilitate the comparison between them: the wheelset speed is $120 \mathrm{~km} / \mathrm{h}$; the excitation wavelength is $1 / 18^{\text {th }}$ of the wheel circumference, and the excitation amplitude is $0.01 \mathrm{~mm}$. The contact parameters being investigated include the nine contact responses used as inputs to the KTH wear model, as well as the instantaneous wear depth calculated by the KTH wear model. 


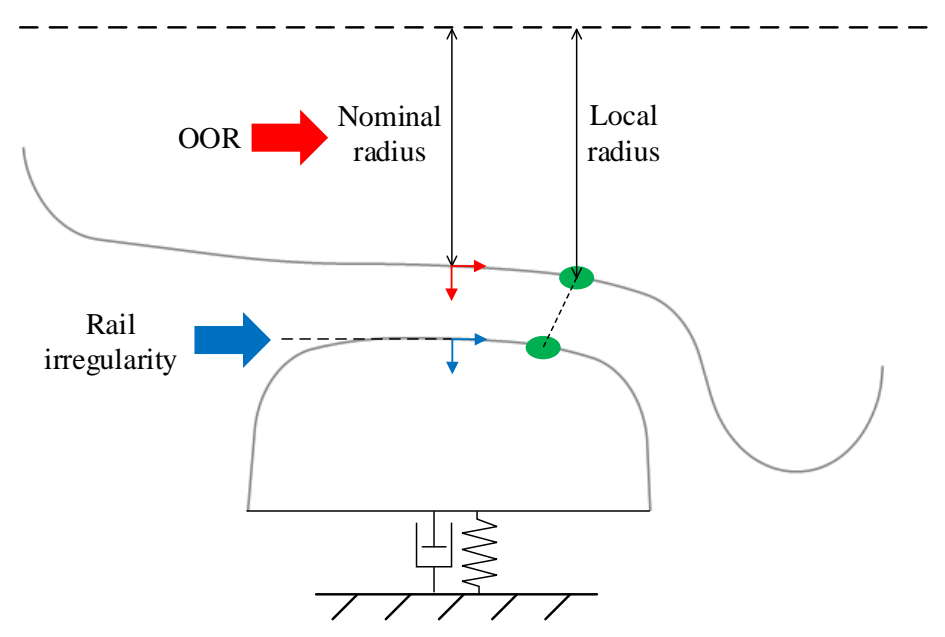

Figure 3. Excitation position of OOR and rail irregularity

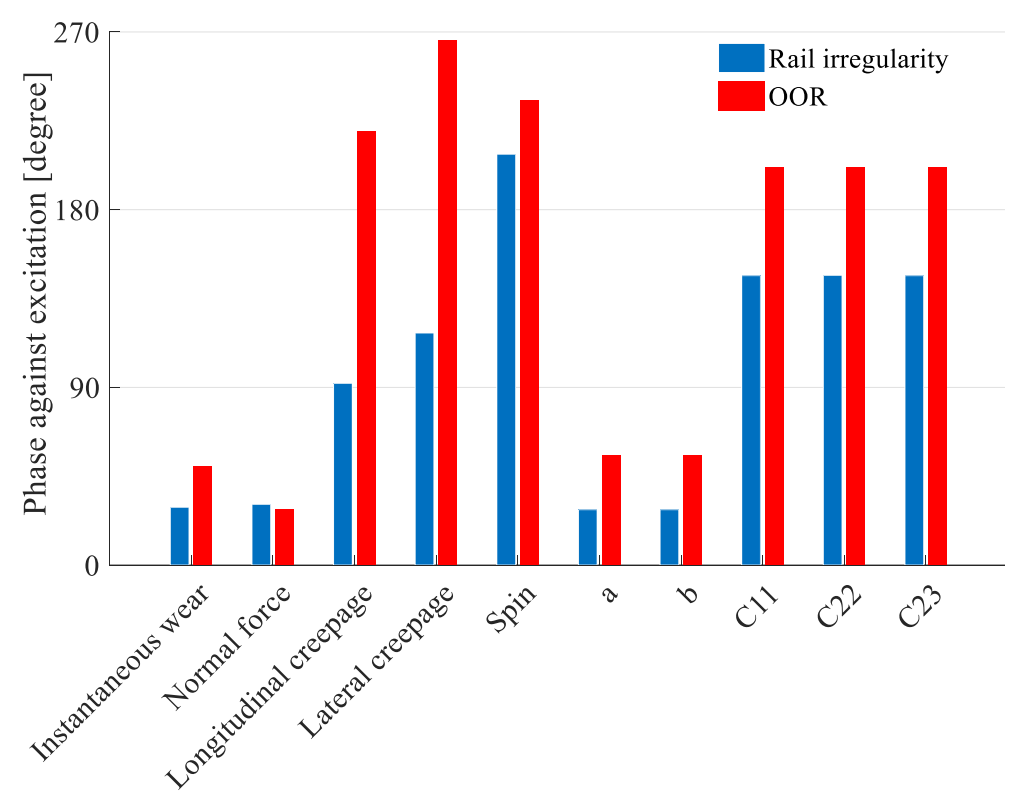

Figure 4. Phase of contact parameters against excitation

Two items of information can be obtained from Figure 4. First, the contact responses can present different phases relative to each other. More simulations show that the phase between contact responses is scenario dependent, and it may not be interpreted as a fixed-relationship. Second, the phase of contact responses caused by OOR and vertical rail irregularity respectively is also different. As a result, the phase of the instantaneous wear depth caused by OOR is different from that by vertical rail irregularity. However, in some self-developed 2D MBS packages [20], the OOR and vertical rail irregularity are both applied to the contact penetration directly as a simplification. With this simplification, the effect of OOR and vertical rail irregularity is exactly the same, and the phase between wear and excitation can be analytically derived. Actually in a more realistic situation, the contact penetration should be a function of the excitation, but not a direct input as an excitation. This implies that the application of the excitation (OOR or rail irregularity) in the simulation has a determining influence on the results, given that the phase of instantaneous wear with respect to initial OOR is the key indicator (see Section 3.1). 


\subsection{The evolution tendency curve}

Before introducing the 'evolution tendency' curve, the instantaneous wear FRF that demonstrates the response of the instantaneous wear against excitation for a wide range of frequencies has to be prepared. It can be drawn by sweeping the wheel velocity with a fixed-wavelength excitation (vertical rail irregularity or initial OOR), and then extracting the amplitude and the phase of the instantaneous wear fluctuation with respect to the excitation. It is worth noting that this fixed wavelength must be exactly divided by the wheel circumference, otherwise the calculated instantaneous wear is not periodic for each wheel revolution. In this case: the fixed wavelength of excitation is one fifth of the wheel circumference; the amplitude of excitation is $10 \mu \mathrm{m}$; and the wheelset speed is swept to get the frequency response up to $500 \mathrm{~Hz}$. The initial OOR and the vertical rail irregularity are applied as excitation respectively with the same settings to allow a comparison between them. The obtained instantaneous wear FRF is shown in Figure 5.
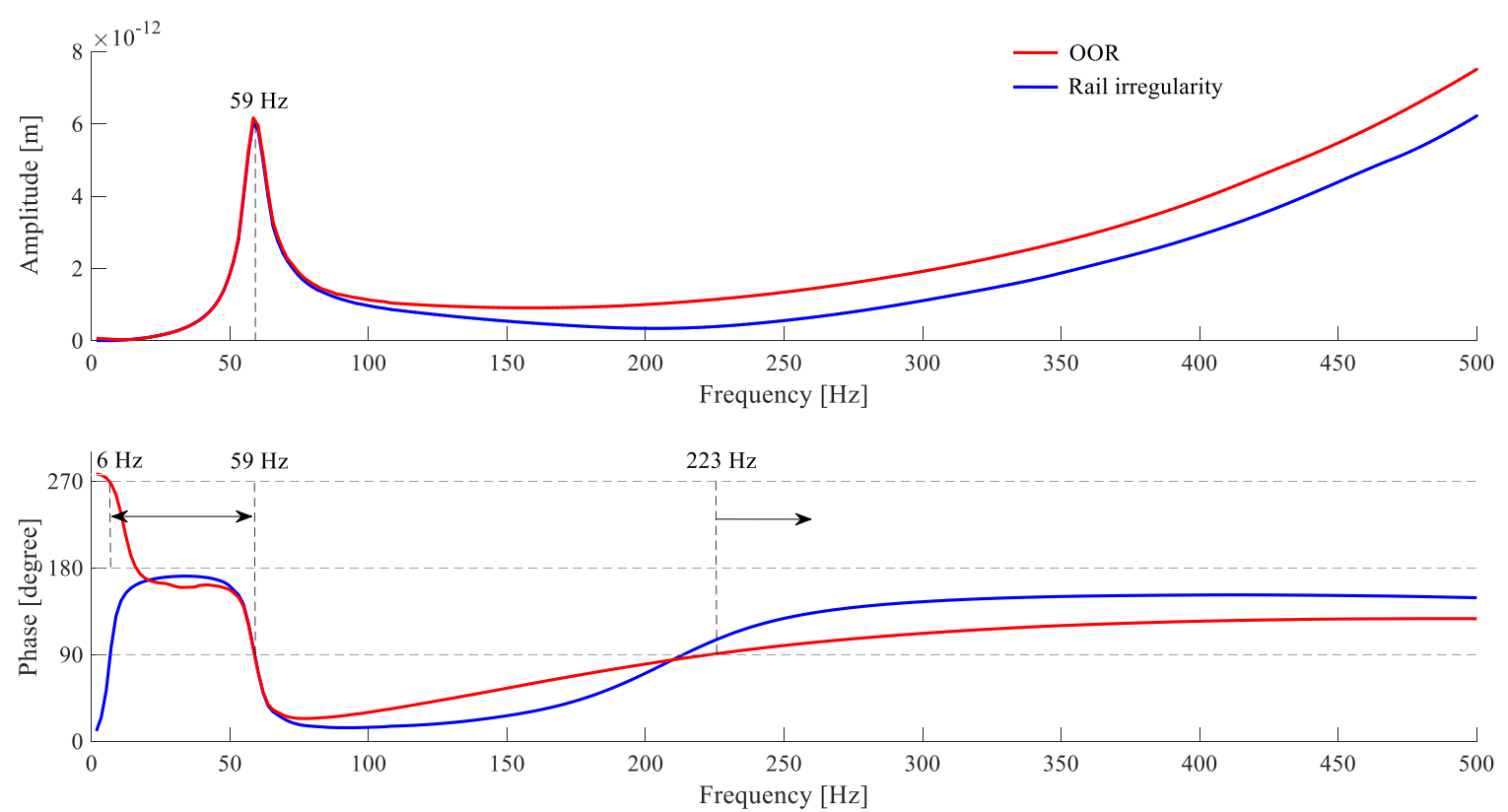

Figure 5. Instantaneous wear FRF

As can be seen in Figure 5, the amplitude spectrum of the OOR and the vertical rail irregularity are similar compared to each other, with their discrepancy gradually becoming bigger for higher frequencies. This implies that the OOR and the vertical rail irregularity have a similar effect in terms of the excitation amplitude, which is reasonable. However, the phase spectrum between them can be more different from each other, although the overall trend is similar. Despite this, the most important aspect is that the phase spectrum of the OOR can be used to identify the frequency range in which the initial OOR tends to grow or diminish. As has been discussed in Section 3.1, the initial OOR will grow when the phase between the instantaneous wear depth and the initial OOR is within 90 to 270 degree. Based on this rule, the frequency range within which the initial OOR tends to grow can be marked in Figure 5, and is from $6 \mathrm{~Hz}$ to $59 \mathrm{~Hz}$ and above $223 \mathrm{~Hz}$, in this case. The $59 \mathrm{~Hz}$ peak is the resonant frequency of the vertical mode of wheelset \& sleeper against the ground, which can be approximately deemed as the $\mathrm{P} 2$ resonance frequency. This frequency is important as it is not only the most dominant resonant frequency in the amplitude spectrum, but also the turning point at 90 degree in the phase spectrum. This frequency can be simply 
estimated by Equation (4) with parameters listed in Table 1. While it is not clear what determines the $6 \mathrm{~Hz}$ and the $223 \mathrm{~Hz}$. Note that the $6 \mathrm{~Hz}$ will be ignored in the following discussion.

$$
f=\sqrt{(\text { sleeper vertical stiffness }) /(\text { wheelset mass+sleeper mass })}=(\sqrt{5.4 \mathrm{e} 8 /(3562+308)}) / 2 \mathrm{pi} \approx 59 \mathrm{~Hz}
$$

Given the instantaneous wear FRF, the amplitude and phase spectrum of it can be further combined to obtain an 'evolution tendency' curve that can indicate both the evolving direction (to grow or to diminish) and the evolution rate of the initial OOR over a range of frequencies in a single curve. Note that in Figure 2 (the upper sub-figure) the growth ratio is a cosine function of the phase between the initial OOR and the instantaneous wear. By shifting this cosine function down by 1 , to set 0 as the boundary line judging the evolution direction (to grow or to diminish), which becomes like $y=-\cos (x)$, and utilizing both information from the amplitude and phase spectrum of the instantaneous wear FRF, the evolution tendency curve can be plotted using Equation (5), which is shown in Figure 6.

$$
\text { Evolution tendency }=-\cos (\text { Phase }) \times \text { Amplitude }
$$

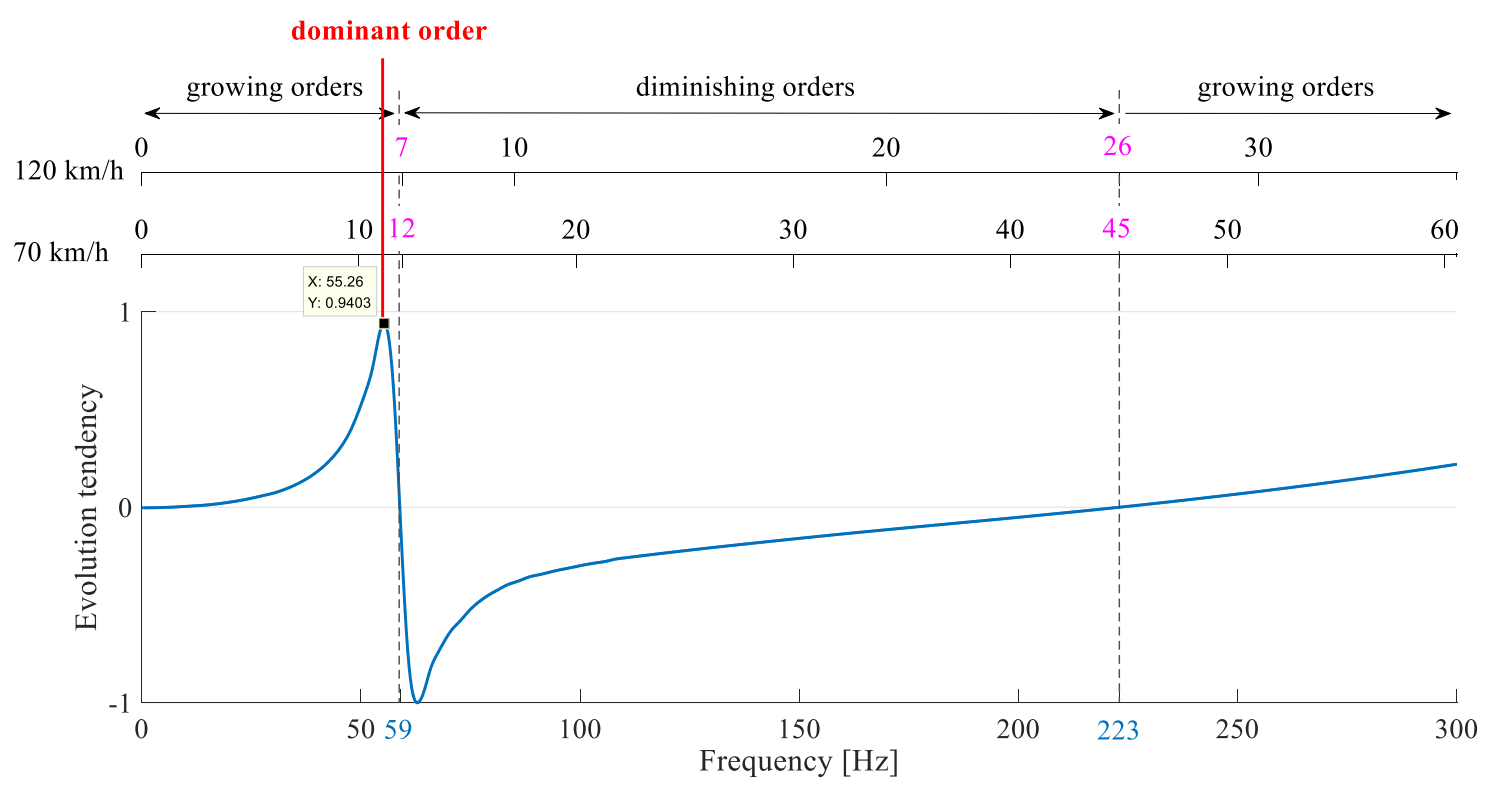

Figure 6. Evolution tendency curve and corresponding growing and diminishing orders at speeds of $70 \mathrm{~km} / \mathrm{h}$ and $120 \mathrm{~km} / \mathrm{h}$

Note that the evolution tendency curve is normalized to be located between -1 and 1 , as its specific value is meaningless and its significance only lies in the comparison between different frequencies. If the evolution tendency is above 0 , the initial OOR tends to grow on its own, otherwise the initial OOR will diminish on its own. Higher absolute value of the evolution tendency means faster evolution speed. Based on this curve, the orders corresponding to the frequencies at speeds of $70 \mathrm{~km} / \mathrm{h}$ and $120 \mathrm{~km} / \mathrm{h}$, as two examples, are plotted in the same figure according to Equation (1), which is shown in the upper part of Figure 6. To facilitate the description, the frequencies at which the evolution tendency turns its direction are called 'boundary frequencies'; the order corresponding to the 'boundary frequency' is called 'boundary order'; the orders that will grow on their own are called 'growing orders'; and the orders that will diminish on their own are called 'diminishing orders'. In Figure 6: $59 \mathrm{~Hz}$ and $223 \mathrm{~Hz}$ are two boundary frequencies, which are marked as blue numbers; the $12^{\text {th }}$ and $45^{\text {th }}$ orders are two boundary orders at $70 \mathrm{~km} / \mathrm{h}$, and the $7^{\text {th }}$ and $26^{\text {th }}$ orders are two boundary orders at $120 \mathrm{~km} / \mathrm{h}$, which are marked as pink numbers. For speed of $70 \mathrm{~km} / \mathrm{h}$, the orders below 12 are growing orders, and those from 12 to 45 
are diminishing orders, and so on. With this figure, the order that would grow the most dominantly on its own can be identified, which is the $11^{\text {th }}$ order at $70 \mathrm{~km} / \mathrm{h}$ and the $6^{\text {th }}$ order at $120 \mathrm{~km} / \mathrm{h}$.

To verify the above rule, the OOR development in the presence of a measured initial OOR is simulated to check which order will grow or diminish as well as their evolution speed, which is shown in Figure 7. Here no rail irregularity is applied to avoid interference. The parameters used are as follows: the speed is $70 \mathrm{~km} / \mathrm{h}$; the number of iterations is 10; the number of wheel revolutions for each iteration is five (although one is enough); the amplification coefficient is $1 \times 10^{6}$ for each iteration; the orders above 40 are filtered to exclude high-frequency components, so orders above 45 (corresponding to $223 \mathrm{~Hz}$ ) will not be presented in the figure. As can be seen from Figure 7, the orders of 12 and below grow while those of 13 and above diminish, with the green line denoting the transition, which corresponds well to the information in Figure 6. The order position at around 12.2 is corresponding to $59 \mathrm{~Hz}$ as the boundary in this case. It can be also found that there is a good consistency in terms of the evolution speed (either growth or diminishment) for all the orders between Figure 6 and Figure 7. Especially, although the roughness of the $11^{\text {th }}$ order is rather low compared to that of other orders at the beginning, it eventually becomes the most dominant order after several evolutions just as Figure 6 predicts. Through this verification, it can be concluded that the evolution tendency curve is a useful tool to predict the evolution of OOR.
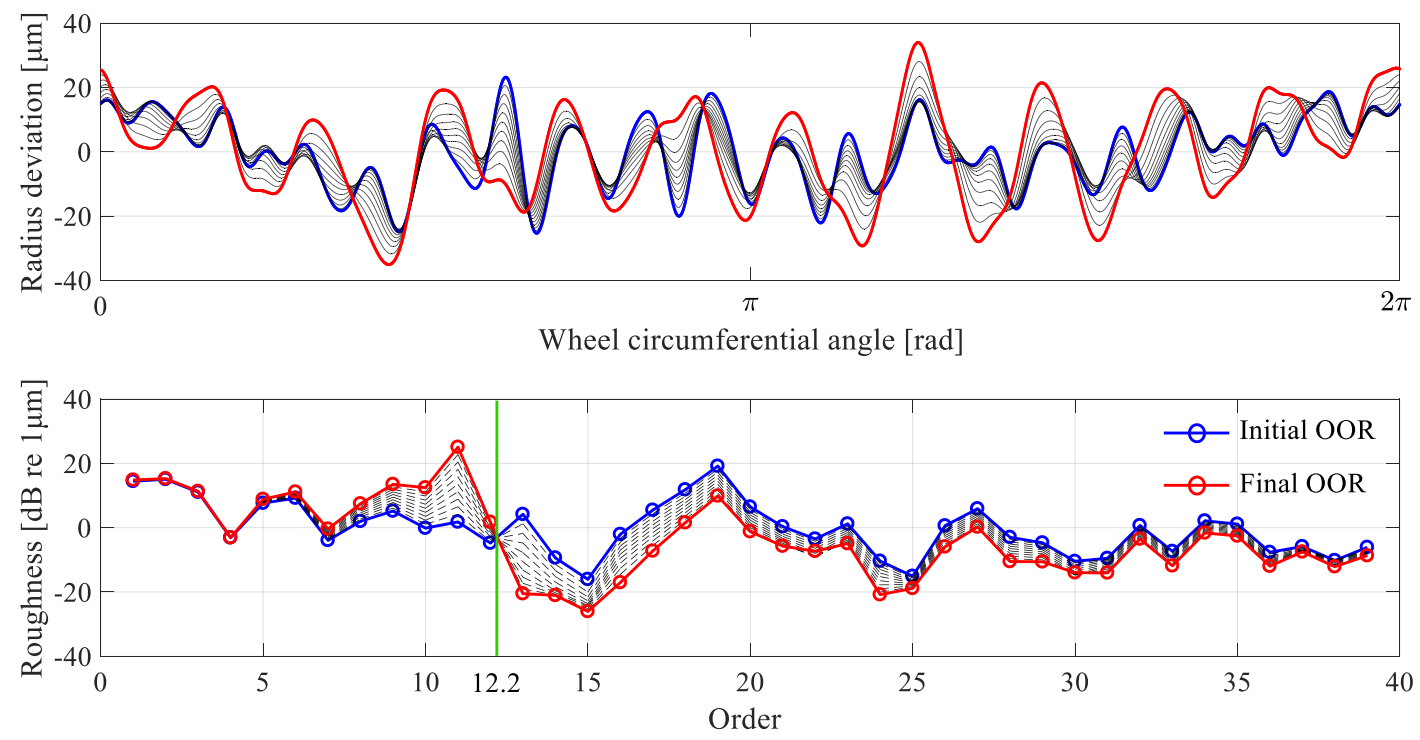

Figure 7. Evolution of OOR due to initial measured OOR

\subsection{Convergency analysis on wheel revolutions}

As shown in Figure 1, for step 3 of the simulation workflow for railway wheel polygonization, the circumferential wear depth is accumulated and averaged by a number of wheel revolutions to get the polygonal wear around the wheel circumference for one iteration. The number of wheel revolutions required for a convergent result is scenario dependent, which will be discussed for the case of harmonic excitation and random excitation respectively.

\subsubsection{The case of harmonic excitation}

For excitation of rail irregularity the wavelength could be arbitrary in reality. If the wheel circumference can be exactly divided by the wavelength, the instantaneous wear is periodic for every wheel revolution. But if the wheel circumference cannot be exactly divided by the wavelength, or the wavelength is bigger than the wheel circumference, the instantaneous wear depth will be different at any point on the wheel circumference for 
consecutive wheel revolutions. Even so, for a harmonic case, the instantaneous wear will always be repeated for a running distance of some wheel revolutions theoretically, no matter what the wavelength is. The minimum number of wheel revolutions for the occurrence of repetition of instantaneous wear can be analytically identified by a uniform Equation (6).

$$
L=\frac{n}{m} C
$$

Where: $L$ is the wavelength of rail irregularity, $C$ is the wheel circumference, and $m$ and $n$ are two natural numbers. By ensuring $m$ and $n$ are relatively prime for each other, $n$ is the minimum number of revolutions for the occurrence of repetition of instantaneous wear. Some representative examples are presented below:

$$
L=\left\{\begin{array}{c}
(1 / 5) C \\
(1 / 5.3) C \\
(1 / 5.74) C \\
(2 / 1) C \\
(3.3 / 1) C
\end{array} \Rightarrow L=\left\{\begin{array}{c}
(1 / 5) C \\
(10 / 53) C \\
(50 / 287) C \\
(2 / 1) C \\
(33 / 10) C
\end{array} \rightarrow n=\left\{\begin{array}{c}
1 \\
10 \\
50 \\
2 \\
33
\end{array}\right.\right.\right.
$$

If $n=1$, one wheel revolution is enough for one iteration as the circumferential wear will be repeated for every wheel revolution. If $n \neq 1$, the minimum number of circles for one iteration should be set to the multiple of $n$, by which the fluctuation of the averaged instantaneous wear depth will remain at a very low level, as the instantaneous wear of each wheel revolution will be just counteracted by each other. This can be checked with Figure 8 as an example $(L=C / 5.3)$. The standard deviation, as a statistical index reflecting the degree of dispersion of a data set, is used to indicate the fluctuating extent of the averaged instantaneous wear depth for specified wheel revolutions (x-coordinate). As can be seen, the standard deviation of the averaged instantaneous wear depth is very small for any wheel revolutions that are multiple of 10, which meets Equation (6) very well, and decreases gradually with the increase of wheel circles.

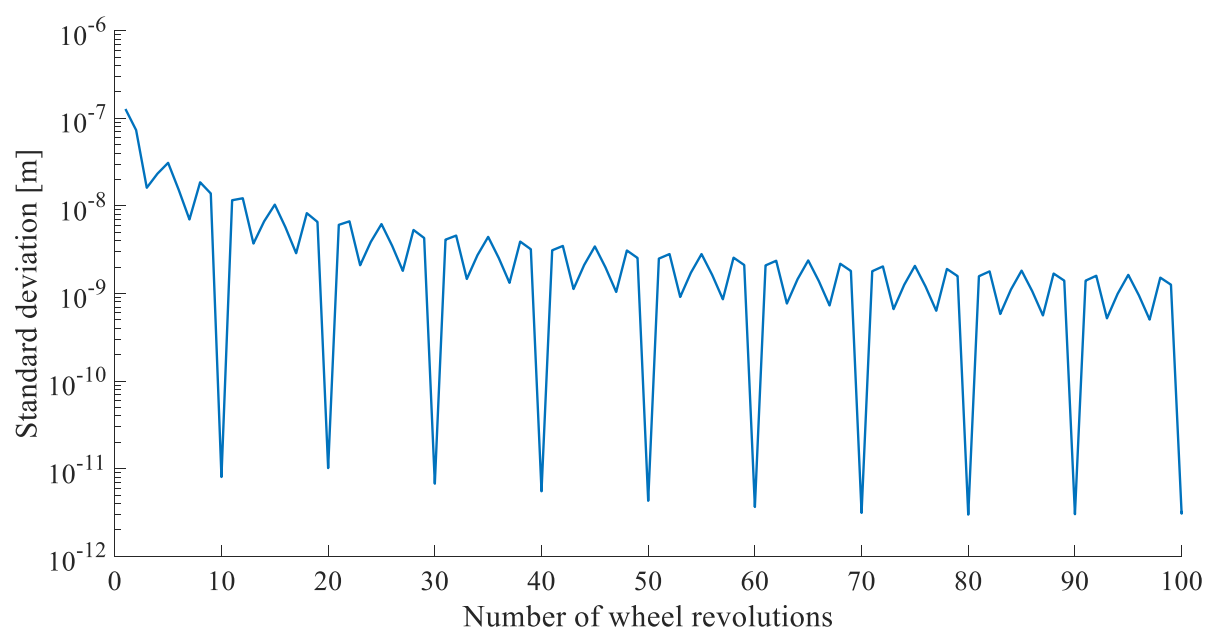

Figure 8. Standard deviation of averaged instantaneous wear depth for specified wheel revolutions $(L=C / 5.3)$

This analysis implies that any rail irregularity with wavelength that cannot exactly divide into the wheel circumference cannot form an OOR easily. A typical example in reality is the sleeper passing oscillation. Although the sleeper passing vibration is a constant excitation with a fixed wavelength, which seems conform to a fixedwavelength mechanism, however if the sleeper distance cannot exactly divide into the wheel circumference, it is not easy for a corresponding OOR to develop. Even the sleeper distance happens to exactly divide into the wheel 
circumference, but the wheel radius will always decrease gradually due to wear, so that the situation of exact division cannot last for a very long time. This could be a factor as to why in reality the OOR corresponding to the sleeper passing frequency is normally not the most dominant one.

In addition, for the case of initial OOR as the excitation on itself, the maximum wavelength of OOR is the wheel circumference itself, and it is not likely for those wavelengths that cannot exactly divide into $C$ to develop significantly, as the OOR wavelength must be continuously smooth around the wheel circumference. In the simulation, the newly updated OOR will always be ensured to be continues around the wheel circumference with the modified Fourier Series method, as stated in Section 2.2. Obviously, the instantaneous wear depth caused by the initial OOR is strictly periodic for every wheel revolution, and only one circle is necessary for this circumstance.

\subsubsection{The case of random excitation}

Under excitation due to random rail irregularity, the instantaneous wear depth is naturally random for every wheel revolution. In this case, the running distance should be long enough to get a convergent result of the averaged instantaneous wear depth for one iteration. A standard track irregularity spectrum FRA 5 (the 5th class track defined by Federal Railroad Administration of US) [21] is adopted to carry out a convergency analysis on the number of wheel revolutions under excitation of random rail irregularity, which is presented in Figure 9. It can be seen that the standard deviation of the averaged instantaneous wear depth becomes convergent after 1000 wheel turns. This implies that if the number of wheel revolutions is not specified to be large enough in the case of random rail irregularity, some significant errors may be introduced.

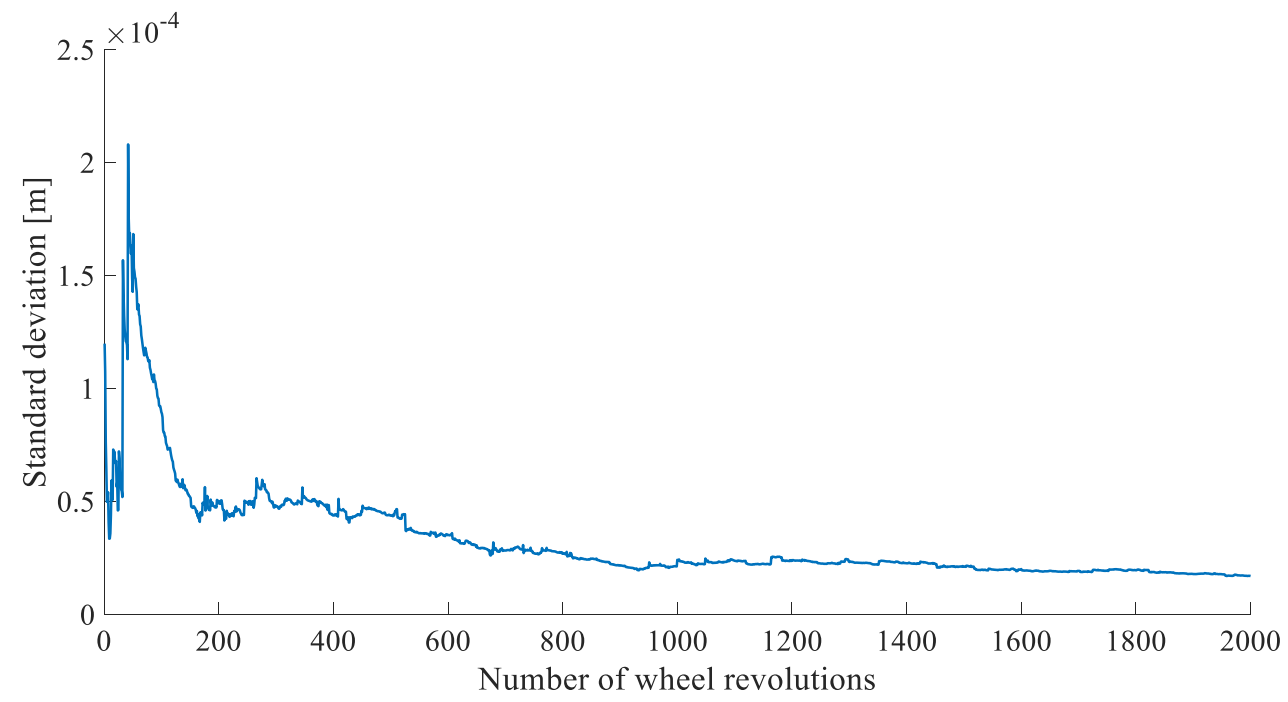

Figure 9. Convergency analysis on the number of wheel revolutions under excitation of random rail irregularity

(FRA 5)

\subsection{Linearity of excitation}

It is found that the fluctuation of the instantaneous wear depth caused by OOR and rail irregularity simultaneously is a simple linear sum of those caused by OOR and rail irregularity individually in the simulation, even though the dynamic system contains non-linear elements. An example of random case is presented here. The initial OOR is configured with the measured data, and white noise is applied to represent the vertical rail irregularity. The result is shown in Figure 10. The solid blue curve is the fluctuation of the instantaneous wear depth caused by vertical rail irregularity of white noise individually, the solid red curve is that caused by initial OOR individually, and the 
solid green curve is that caused by vertical rail irregularity and initial OOR simultaneously. The dashed black curve is the sum of solid blue curve and solid red curve. As can be seen, the solid green curve matches the dashed black curve perfectly. If a random case is proven, let alone a harmonic case. More simulations show that the linearity of excitation also applies for various combination of OOR and rail irregularity, which means that the overall result of the instantaneous wear depth caused by OOR and/or rail irregularity with multiple frequency components is the linear superposition of those caused by OOR and/or rail irregularity with single harmonic frequency. This characteristic is important to aid the understanding of more complex scenarios.

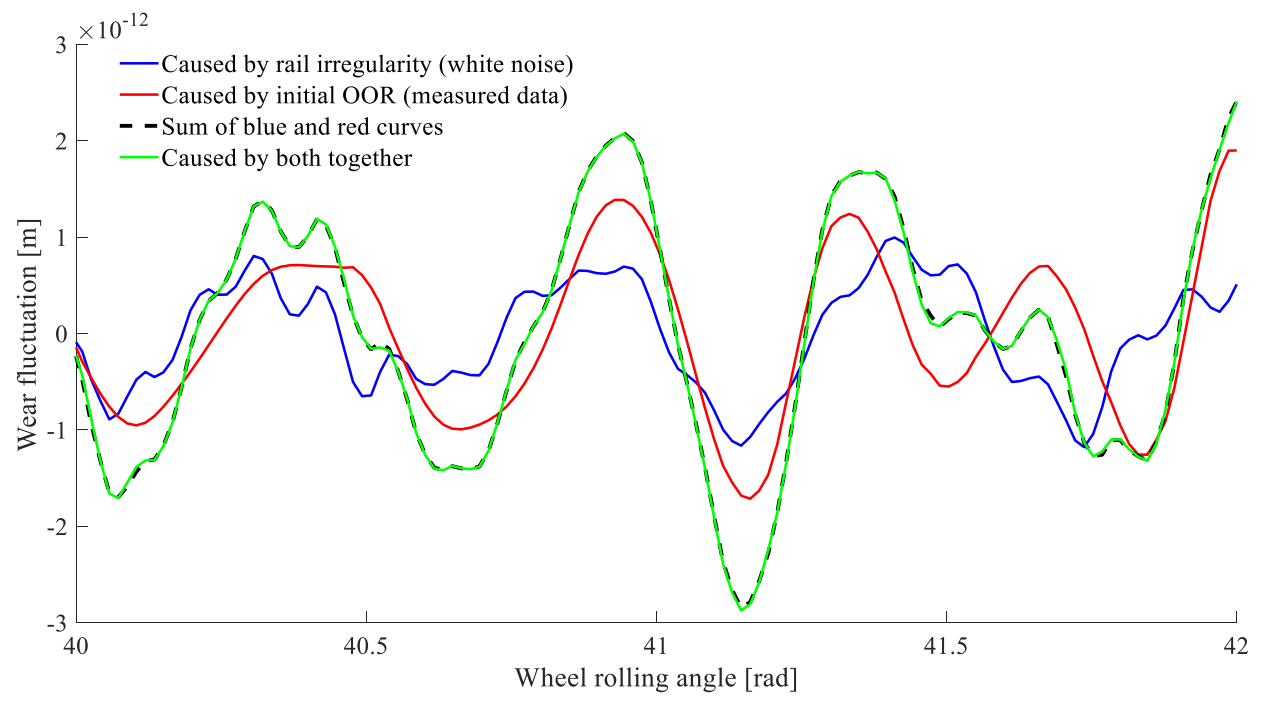

Figure 10. Linearity analysis for instantaneous wear fluctuation caused by vertical rail irregularity of white noise and measured OOR

\subsection{Contribution of newly developed OOR}

Considering the linearity of excitation, the iterative process for OOR evolution can be presented in Figure 11. In this figure: EOT is the abbreviation of Excitation Of Track, and OOR means wheel Out-Of-Roundness; $W_{\text {EOT }}$ is the fluctuation of polygonal wear contributed by EOT individually (dashed blue arrows), $W_{\mathrm{OOR}}$ is the fluctuation of polygonal wear contributed by OOR individually (dashed red arrows), and $W_{\text {TOTAL }}$ is the sum of them amplified by a large coefficient $k$ (assuming the same value for every iteration). All the superscripts indicate the iteration step; OOR ${ }^{0}$ is the initial OOR. For the $i^{\text {th }}$ iteration, the OOR is updated by: $\mathrm{OOR}^{i}=\mathrm{OOR}^{i-1}-W_{\mathrm{TOTAL}}^{i}$. With $n$ iterations the OOR evolution is obtained. 


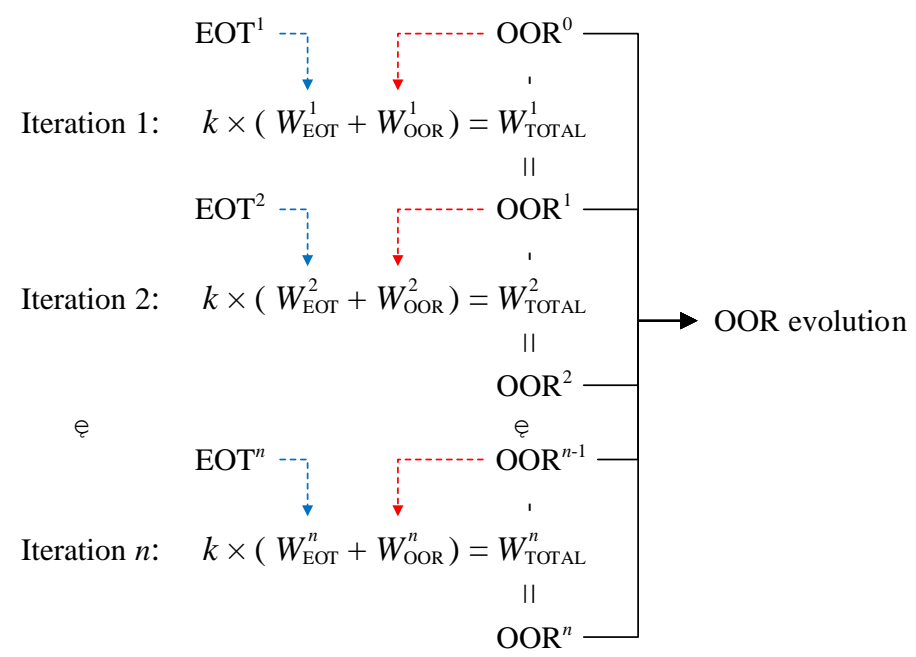

Figure 11. Iterative process for OOR evolution

As can been seen from Figure 11, the wheel OOR is gradually evolving with contribution of both track excitation and newly developed OOR. If track excitation is the same for the whole iteration process, the $W_{\text {ЕOT }}$ is actually the same for each iteration. In this case, the OOR evolution contributed by track excitation is the linear sum of each

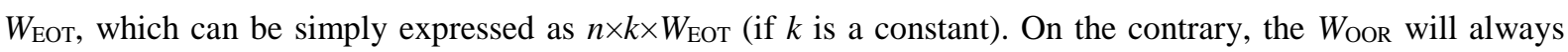
change along with the iterations due to introduction of newly developed OOR, and the contribution of $W_{\mathrm{OOR}}$ to the OOR evolution could be positive or negative depending on the range of excitation frequency (see Section 3.3). An example is presented in Figure 12 to demonstrate the negative effect of newly developed OOR on OOR growth. In this example: only vertical rail irregularity with a harmonic wavelength $(C / 13)$ is applied as the track excitation persisting for the whole iteration process; the fluctuation amplitude of rail irregularity is $10 \mu \mathrm{m}$; $k$ is $1 \times 10^{6}$ as a constant; the number of iterations $(n)$ is 10 , the speed is $70 \mathrm{~km} / \mathrm{h}$. In Figure 12, the dashed blue curve is the imaginary final OOR that would evolve to $31.15 \mathrm{~dB}$ caused by rail irregularity alone, without contribution from newly developed OOR; the solid red curve is the actual final OOR that evolves due to both rail irregularity and newly developed OOR, with nine dashed black curves indicating the OOR at discrete steps during iterative process. It is obvious that the newly developed OOR plays a negative role in resisting the OOR growth in this case. This is because the $13^{\text {th }}$ order is a diminishing order at a speed of $70 \mathrm{~km} / \mathrm{h}$, as shown in Figure 6 .

Having a closer look at Figure 12, after around five iterations when the radius deviation approaches $10 \mu \mathrm{m}$ (the amplitude of rail irregularity) corresponding to the roughness of $20 \mathrm{~dB}$, the OOR growth stops for further iterations. This is because when the newly developed OOR has reached the same roughness level of the rail irregularity, the instantaneous wear depth caused by them respectively is comparable to each other, and thus will be counteracted. This is an interesting phenomenon as it implies that the roughness level of the OOR will not exceed that of the rail irregularity for diminishing orders, in the given simulation case. 


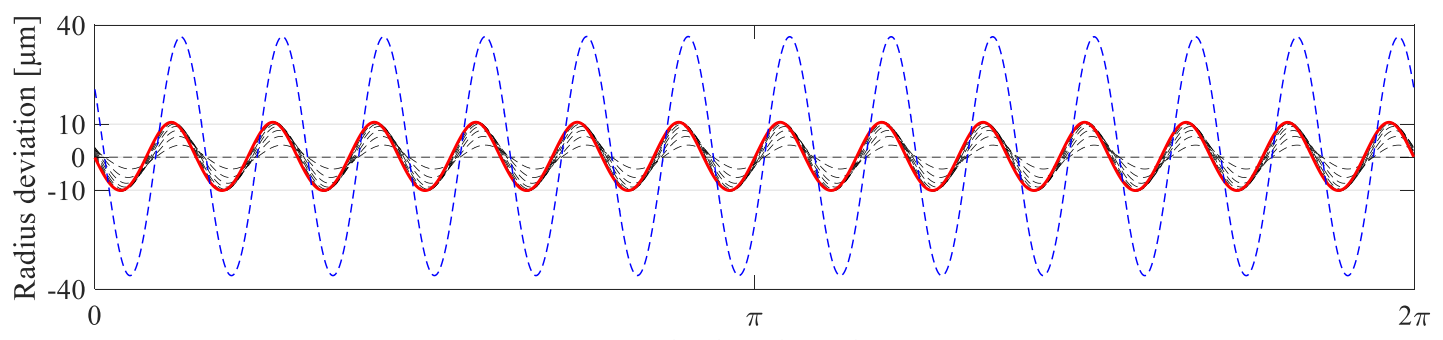

Wheel angle [rad]

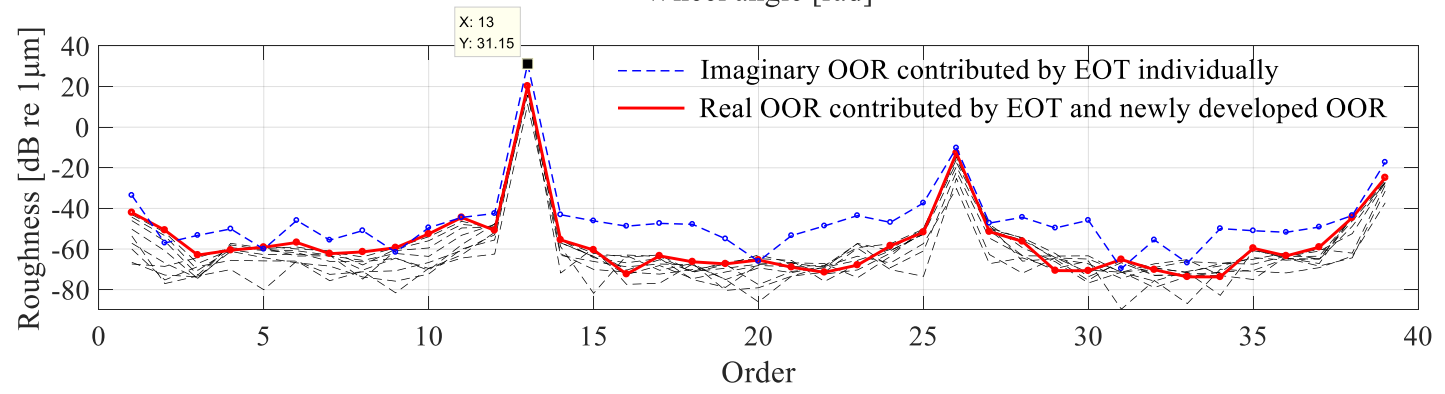

Figure 12 . The negative effect of newly developed OOR on OOR growth (the $13^{\text {th }}$ Order)

\subsection{Contribution of track excitation}

Continuing the above section, the simulation is further carried out with consideration of track excitation including both the random track irregularity and the sleeper passing oscillation. Most parameters are kept the same as above, with some additional modifications being added: the random track irregularity is FRA 5; the sleeper distance is $0.6 \mathrm{~m}$; a same initial OOR in Figure 7 is introduced but multiplied by 0.01 to reduce its weight; the amplification coefficient for each iteration is decreased from $1 \times 10^{6}$ to $1 \times 10^{4}$ to avoid contact detachment during the iteration; the wheel revolutions for one iteration is 1000 . The sample of the random track irregularity is set to be uncorrelated for each iteration to avoid repetitive scenarios. The OOR evolution under excitation of only vertical FRA 5 and full FRA 5 consisting of vertical, lateral, rolling, and gauge irregularities, are presented in Figure 13 and Figure 14 respectively.
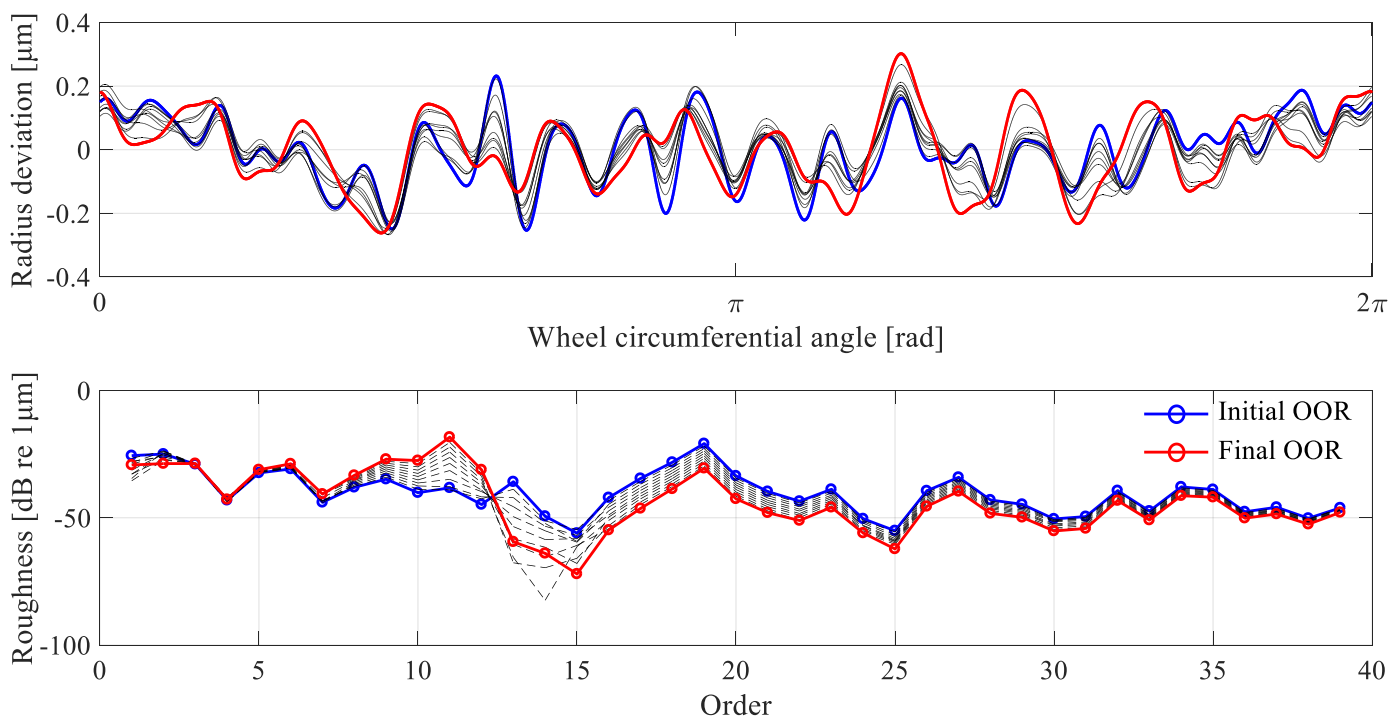

Figure 13. OOR evolution under excitation of only vertical random rail irregularity (FRA 5) 


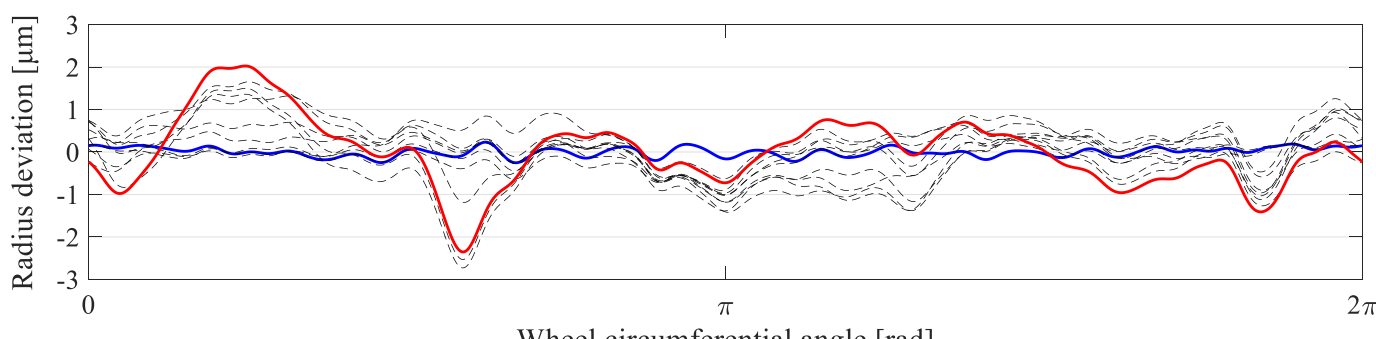

Wheel circumferential angle [rad]

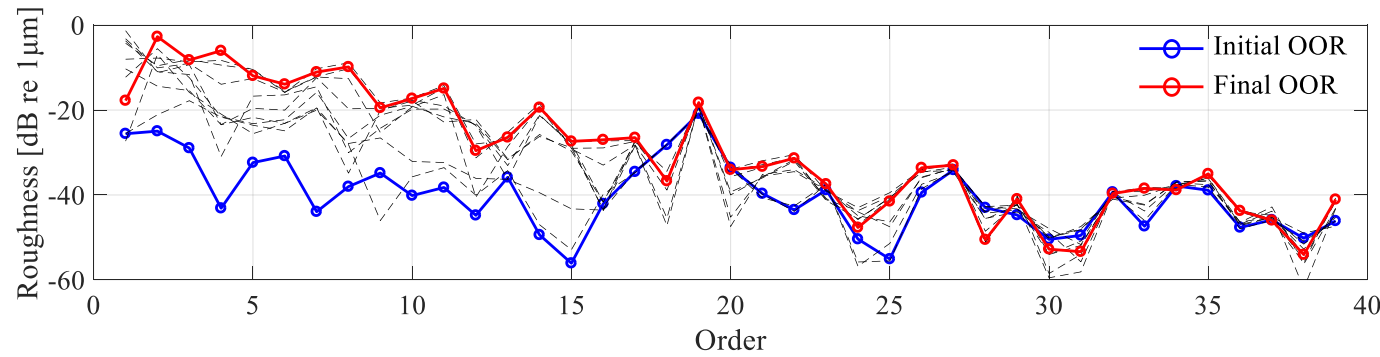

Figure 14. OOR evolution under excitation of full random rail irregularity (FRA 5)

As can be seen from Figure 13, if only the vertical track irregularity is applied, the evolution trend is similar to the case of only initial OOR without track excitation (see Figure 7), implying that the contribution of the vertical random track irregularity to the OOR evolution is negligible compared to that of initial OOR. This is because the standard deviation of the averaged polygonal wear caused by random rail irregularity for a large number of wheel revolutions (1000 in this case) is convergent to a small amount, which has been discussed in Section 3.4.2. In this situation, the OOR evolution is still driven by the evolution tendency curve, with the $11^{\text {th }}$ order growing the most dominantly. This is a further confirmation of the effectiveness of the evolution tendency curve. In addition, the sleeper passing frequency does not produce an obvious OOR $(3.925 / 0.6 \approx 6.5)$ to develop because the wheel circumference cannot be exactly divided by the sleeper distance, and the number of wheel revolutions (1000) is large enough for the polygonal wear caused by sleeper passing oscillation to be counteracted among a large number of wheel revolutions, which has also been discussed in Section 3.4.1.

However, under the excitation of full FRA 5, the result of OOR evolution changes dramatically, as shown in Figure 14. As more excitation energy is contained in the full track irregularity and the main energy is located within the lower frequencies (below $20 \mathrm{~Hz}$ ), the lower orders of OOR can develop more significantly than other orders. In this situation the contribution of full track irregularity plays a dominant role for the OOR evolution, and the contribution of initial and newly developed OOR can be covered up. Note that the evolution tendency curve mentioned above is obtained only for the case of excitation in vertical direction (vertical rail irregularity or initial OOR), if other types of track irregularity (lateral, rolling, and gauge irregularities) are taken into consideration, the instantaneous wear FRFs for excitation of different types have to be obtained respectively so as to derive the corresponding evolution tendency curves which could be very different from each other. These evolution tendency curves should be synthetically utilised to establish a more instructive indicator to predict the OOR evolution under excitation of full random track irregularities, which however is not contradict to the basic validity of evolution tendency curve for OOR prediction. This is a future work. 


\section{The general conditions for OOR to evolve}

Based on the rules discussed above and previous research by the authors [12,16,22], the general conditions for OOR to evolve are proposed as follows:

(1) The wheel is running at a constant speed for a long time to provide a stable environment for the OOR to develop gradually.

(2) One or more structural modes can be significantly excited leading to a persisting vibration with fixed frequency to the wheel/rail interface for a long time.

(3) The energy distribution of the track excitation allows some structural modes to be excited dominantly.

(4) The OOR order that would grow the most dominantly at a given speed can be identified by the evolution tendency curve obtained from the instantaneous wear FRF.

\section{Discussions}

\subsection{Assumptions}

In this paper, the wheel radius is assumed not to change during the iterations. However, as the wheel radius is an important factor that can determine the number of OOR orders, the change of wheel radius should be considered in the future. If so, the mean value of the instantaneous wear depth should be considered as it will determine the reduction speed of the wheel radius. In addition, the wheel transverse profile is also assumed not to change. Although this limitation could be broken through with more complex program, it is believed that the change of transverse profile might has less importance on the wheel polygonization, but of course it should be confirmed in the future.

\subsection{Instantaneous wear FRF}

The instantaneous wear FRF is a very important indicator to identify the potential OOR orders that would develop dominantly. Especially the phase spectrum of the instantaneous wear FRF is the key indicator judging the 'growing orders' and 'diminishing orders'. However, the phase between the instantaneous wear and excitation is rather hard to be interpreted. There are two phase relationships: one is the phase between the excitation and the contact responses, the other is the phase between the contact responses and the instantaneous wear. Both of them present as a complicated issue individually, which has to be related to the modeling method. It seems that different modeling methods will directly lead to different results, which implies that at least some models may not be sufficiently realistic to faithfully predict OOR evolution. However, this does not detract from the fundamental observations made here. If the modeling method in terms of the two phase initiation mechanism can be improved to match the reality, the evolution tendency curve obtained from the instantaneous wear FRF could be more significant as a guidance.

\subsection{Conditions for wheel OOR to develop}

The most possible root cause currently suspected in academic community are pointing to the wheelset flexibility and the rail flexibility, although other component modes could also be the causes. No matter what the cause is, the common rule is that the structural mode must dominate the evolution tendency curve, so that a corresponding OOR 
order can develop dominantly. In addition, in reality the conditions of (2) and (3) mentioned in Section 4 cannot easily be confirmed as scenarios can vary significantly. For example, some structural modes can be dominantly excited in some sections of the track but not in other sections; some types of vehicle can have serious OOR issue on one track, but other types of vehicle may not. It is believed that a good match between the track and the vehicle is the best solution to avoid the OOR problem.

\section{Conclusion}

This paper reports a fundamental research into the general conditions for railway wheel polygonal wear to evolve. A common workflow for prediction of railway wheel polygonization is presented in terms of some assumptions, a simulation scheme, and a uniform wear model. Based on this workflow, some rules for evolution of railway wheel polygonization are proposed providing innovative perspectives to understand the basic mechanism of OOR. After summarizing these rules, the general conditions for railway wheel polygonal wear to evolve are established. Some main points in this paper are listed below.

(1) The phase between the instantaneous wear and the excitation is the key indicator determining the OOR evolution direction (to grow or to diminish).

(2) The evolution tendency curve obtained from the instantaneous wear FRF is proposed as a useful tool to predict the OOR evolution, especially for predicting the OOR order that would grow dominantly at a given speed.

(3) If one or more structural modes can dominate the evolution tendency curve, and the energy distribution of track excitation allows this/these structural modes to be excited effectively, corresponding OOR orders can occur dominantly.

\section{Funding}

The present work is supported by CRRC Zhuzhou Locomotive Co.,Ltd. This support is gratefully acknowledged.

\section{References}

[1] Barke DW, Chiu WK. A Review of the Effects of Out-Of-Round Wheels on Track and Vehicle Components. Proc Inst Mech Eng F: J Rail Rapid Transit. 2005;219(3):151-175.

[2] Tao G, Wen Z, Liang X, et al. An investigation into the mechanism of the out-of-round wheels of metro train and its mitigation measures. Veh Syst Dyn. 2018:1-16.

[3] Johansson A, Andersson C. Out-of-round railway wheels-a study of wheel polygonalization through simulation of three-dimensional wheel-rail interaction and wear. Veh Syst Dyn. 2005;43(8):539-559.

[4] Tao G, Wang L, Wen Z, et al. Experimental investigation into the mechanism of the polygonal wear of electric locomotive wheels. Veh Syst Dyn. 2017:1-17.

[5] Wu X, Rakheja S, Cai W, et al. A study of formation of high order wheel polygonalization. Wear. 2019;424:114.

[6] Meinke P, Meinke S. Polygonalization of wheel treads caused by static and dynamic imbalances. J Sound Vib. 1999 1999/11/11;227(5):979-986.

[7] Shen W, Song C, Li G, et al. Research for high-speed EMU wheel hardness and polygon-form relationships with solutions. Railway Locomotive \& Car. 2018;38(4):18-23.

[8] Ye Y, Shi D, Krause P, et al. Wheel flat can cause or exacerbate wheel polygonization. Veh Syst Dyn. 2019:130. 
[9] Spangenberg U. Variable frequency drive harmonics and interharmonics exciting axle torsional vibration resulting in railway wheel polygonisation. Veh Syst Dyn. 2019:1-21.

[10] Morys B. Enlargement of out-of-round wheel profiles on high speed trains. J Sound Vib. 1999 1999/11/11;227(5):965-978.

[11] Fu B, Bruni S, Luo S. Numerical simulation for polygonal wear of railway wheels. 11th international conference on contact mechanics and wear of rail/wheel systems (CM2018); Delft, The Netherlands2018.

[12] Peng B, Iwnicki S, Shackleton P, et al. A practical method for simulating the evolution of railway wheel polygonalization. 25th international symposium on dynamics of vehicles on roads and tracks; Rockhampton, Australia2017. p. 753-758.

[13] Tao G, Wang L, Wen Z, et al. Measurement and assessment of out-of-round electric locomotive wheels. Proc Inst Mech Eng F: J Rail Rapid Transit. 2016;232(1):275-287.

[14] Johansson A. Out-of-round railway wheels - assessment of wheel tread irregularities in train traffic. J Sound Vib. 2006 2006/06/13/;293(3):795-806.

[15] Jendel T, Berg M. Prediction of Wheel Profile Wear. Veh Syst Dyn. 2002;37(sup1):502-513.

[16] Peng B, Iwnicki S, Shackleton P, et al. Comparison of wear models for simulation of railway wheel polygonization. Wear. 2019 2019/10/15/;436-437:203010.

[17] Pearce TG, Sherratt ND. Prediction of wheel profile wear. Wear. 1991 1991/04/20;144(1):343-351.

[18] Lewis R, Dwyer-Joyce RS. Wear mechanisms and transitions in railway wheel steels. Proc Inst Mech Eng J: J Engineering Tribology. 2004;218(6):467-478.

[19] Zobory I. Prediction of Wheel/Rail Profile Wear. Veh Syst Dyn. 1997;28(2):221-259.

[20] Liu X, Zhai W. Analysis of vertical dynamic wheel/rail interaction caused by polygonal wheels on high-speed trains. Wear. 2014 2014/06/15/;314(1):282-290.

[21] Garg VK, Dukkipati RV. Dynamics of railway vehicle systems. Toronto, Ont: Academic Press; 1984. (Book, Whole).

[22] Peng B, Iwnicki S, Shackleton P, et al. The influence of wheelset flexibility on polygonal wear of locomotive wheels. Wear. 2019;432-433:102917. 